\title{
Clinical and functional change in multifocal motor neuropathy treated with IVIg
}

\begin{abstract}
We determined the clinical progression, disability and outcome of 11 Multifocal Motor Neuropathy (MMN) patients from Malaysia. Mean patient age was 46.8 (SD 13.3), with mean disease duration of 108.0 months (SD 80.2). All reported unilateral limb weakness at onset. At diagnosis, after mean 49.9 months (SD 73.5) delay, 7 (63.6\%) had more than 2 limbs involvement. Nine (90\%) of 10 patients received induction IVIg dose of $2.0 \mathrm{gm} / \mathrm{kg}$ responded, demonstrated improvement in MRCSS of $>2$ points or $\mathrm{mRS}$ score of $>1$ point. We observed $38.5 \%$ drop in IVIg dose to mean $1.12 \mathrm{gm} / \mathrm{kg} / \mathrm{month}$ after 12 months of treatment, and a further $34.8 \%$ drop upon 24th month treatment to mean dose of 0.73 $\mathrm{gm} / \mathrm{kg} / \mathrm{month}$. This was in parallel with initial improvement in MRCSS and mRS, observed among $88.9 \%$ and $77.8 \%$ of the patients, and later further improvement $(33.3 \%)$ or stabilization $(66.7 \%)$ of $\mathrm{mRS}$ score toward 2nd year. During the same period, $50 \%$ of patients reported deterioration in ONLS, 33.3\% in grip strength and $16.7 \%$ in MMN-RODS. Beyond 36th month, average annual IVIg dose increased at $0.12 \mathrm{gm} / \mathrm{kg} / \mathrm{year}$ (SD 0.09 ) or $11.2 \%$, up to the 84th months. Despite that, progressive deterioration was observed in term of number of limbs involvement, definite motor conduction blocks on electrophysiology study, and both clinical as well as functional scores. Although IVIg dose reduction for maintenance treatment in MMN is recommended, careful clinical assessment is required to prevent under-treatment. Use of reliable and responsive modern outcome measures is important to quantify clinically relevant change to guide therapy.
\end{abstract}

Keyword: Multifocal motor neuropathy; Inflammatory neuropathy; Intravenous immunoglobulin; Clinical outcome; Malaysia; Southeast Asia 